\title{
Integrated petrophysical log characterization for tight carbonate reservoir effectiveness: A case study from the Longgang area, Sichuan Basin, China
}

\author{
Deng Shaogui ${ }^{1,2 *}$, Wang Yang ${ }^{2,3}$, Hu Yunyun ${ }^{2}$, Ge Xinmin² and He Xuquan ${ }^{4}$ \\ ${ }^{1}$ School of Earth Sciences and Resources, China University of Geosciences, Beijing 100083, China \\ ${ }^{2}$ School of Geosciences, China University of Petroleum, Shandong 266580, China \\ ${ }^{3}$ China Petroleum Great Wall Drilling Geological Research Institute, Liaoning 124000, China \\ ${ }^{4}$ China Petroleum Southwest Oil and Gas Field Branch, Sichuan 610051, China \\ (c) China University of Petroleum (Beijing) and Springer-Verlag Berlin Heidelberg 2013
}

\begin{abstract}
Ultra-low porosity and permeability, inhomogeneous fracture distribution, and complex storage space together make the effectiveness evaluation of tight carbonate reservoirs difficult. Aiming at the carbonate reservoirs of the Da'anzhai Formation in the Longgang area of the Sichuan Basin, based on petrophysical experiments and logging response characteristics, we investigated the storage properties of matrix pores and the characteristics of fracture development to establish a method for the characterization of effectiveness of tight reservoirs. Mercury injection and nuclear magnetic resonance (NMR) experiments show that the conventional relationship between porosity and permeability cannot fully reflect the fluid flow behavior in tight matrix pores. Under reservoir conditions, the tight reservoirs still possess certain storage space and permeability, which are controlled by the characteristic structures of the matrix porosity. The degree of fracture development is crucial to the productivity and quality of tight reservoirs. By combining the fracture development similarity of the same type of reservoirs and the fracture development heterogeneity in the same block, a three-level classification method of fracture development was established on the basis of fracture porosity distribution and its cumulative features. According to the actual production data, based on the effectiveness analysis of the matrix pores and fast inversion of fracture parameters from dual laterolog data, we divided the effective reservoirs into three classes: Class I with developed fractures and pores, and high-intermediate productivity; Class II with moderately developed fractures and pores or of fractured type, and intermediate-low productivity; Class III with poorly developed fractures and matrix pores, and extremely low productivity. Accordingly log classification standards were set up. Production data shows that the classification of effective reservoirs is highly consistent with the reservoir productivity level, providing a new approach for the effectiveness evaluation of tight reservoirs.
\end{abstract}

Key words: Matrix porosity, fracture porosity, reservoir effectiveness, reservoir classification, petrophysical log characterization

\section{Introduction}

The combination of matrix pores and fractures determines the storage space type of carbonate reservoirs. Generally the matrix pore development characteristics and the type of pore structure determine the reservoir storage capacity, while fractures serve as both storage space and seepage channels. The degree of fracture development even controls reservoir productivity behavior (Guerriero et al, 2012; Guo et al, 2012; Li et al, 2012; Wang et al, 2011; Xu et al, 2013). The effectiveness of matrix pores is evaluated according

*Corresponding author. email: dengshaogui@126.com

Received November 16, 2012 to the pore structure by utilizing techniques such as core analysis, optical microscopy, image analysis, scanning electron microscopy, and mercury injection capillary pressure, and NMR $T_{2}$ distribution combined with logs (Chekani and Kharrat, 2012; Kim et al, 2011; Kuz'min and Skibitskaya, 2012; Mai and Kantzas, 2007; Marathe et al, 2012; Mohammadlou et al, 2012; Schoenfelder et al, 2008; Tsakiroglou et al, 2009; Westphal et al, 2005).

Fracture evaluation is one of the core tasks in tight carbonate reservoir evaluation (Lamarche et al, 2012). Electric imaging logging cannot only directly show the degree of fracture development and occurrence, but also accurately predict the fracture effectiveness. However, its high cost and thus limited quantity restricts its large-scale application 
in regional logging evaluation. Dual laterolog data is often used to determine the fracture porosity because of its good focusing ability and correlation with the fractures. Sibbit and Faivre (1985) found the relationship between dual laterolog responses and the opening degree of a single horizontal or vertical fracture. Pezard and Anderson (1990) obtained the formulas for calculating the porosity of horizontal and vertical fractures. Li et al (1996) proposed a group of formulas for fast calculation of fracture porosity with three different fracture occurrences by dual laterolog resistivity. Deng et al (2006) identified the characteristics of dual laterolog responses to fractures in fractured tight carbonate reservoirs and established the interpretation methods.

Due to the poor understanding on the fluid storage and flow mechanism in tight carbonate reservoirs, we have no clear ideas of the contribution of different reservoir space to the productivity in tight carbonate reservoirs. Some indicated that the Da'anzhai Formation was a fractured carbonate reservoir, while others thought that all types of pores make important contributions to the reserves and the long-term low and stable production, thus the reservoir was not simply a fractured reservoir (Liang et al, 2011). Therefore, the identification and evaluation of reservoir effectiveness are very important (Bust et al, 2011; Yang et al, 2011; Asgari and Sobhi, 2006). This focuses on the following aspects: 1) whether the matrix pores are the effective storage space or not, which determines the reservoir type and storage property; 2) the development degree and effectiveness of fractures, which are necessary for the low porosity and low permeability reservoir to obtain high production. In this study, on the basis of mercury injection and NMR experiments, we analyzed the matrix pore structures and investigated the fluid flow capacity. Using dual laterolog data, the fracture parameters were quantitatively evaluated in combination with electric imaging logging. The reservoir effectiveness was examined in terms of the matrix pores and the fractures and, combined with production test data, a method for the comprehensive characterization of tight carbonate reservoir effectiveness was explored.

\section{General characteristics of the tight reservoirs of the Da'anzhai Formation}

The Da'anzhai reservoir in the Longgang area, Sichuan Basin deposited in a moderate-deep lake environment mainly consists of interbedded grey shelly limestone and black shale of different thicknesses, in which the storage space is dual pore-fracture porosity, as shown in Fig. 1. The shelly limestone, mainly composed of micrite shelly limestone and shaly shelly limestone, is mainly calcite in mineral composition with slight dolomite, and rich in shell content, generally above $60 \%$. Shaly shelly limestone has a higher

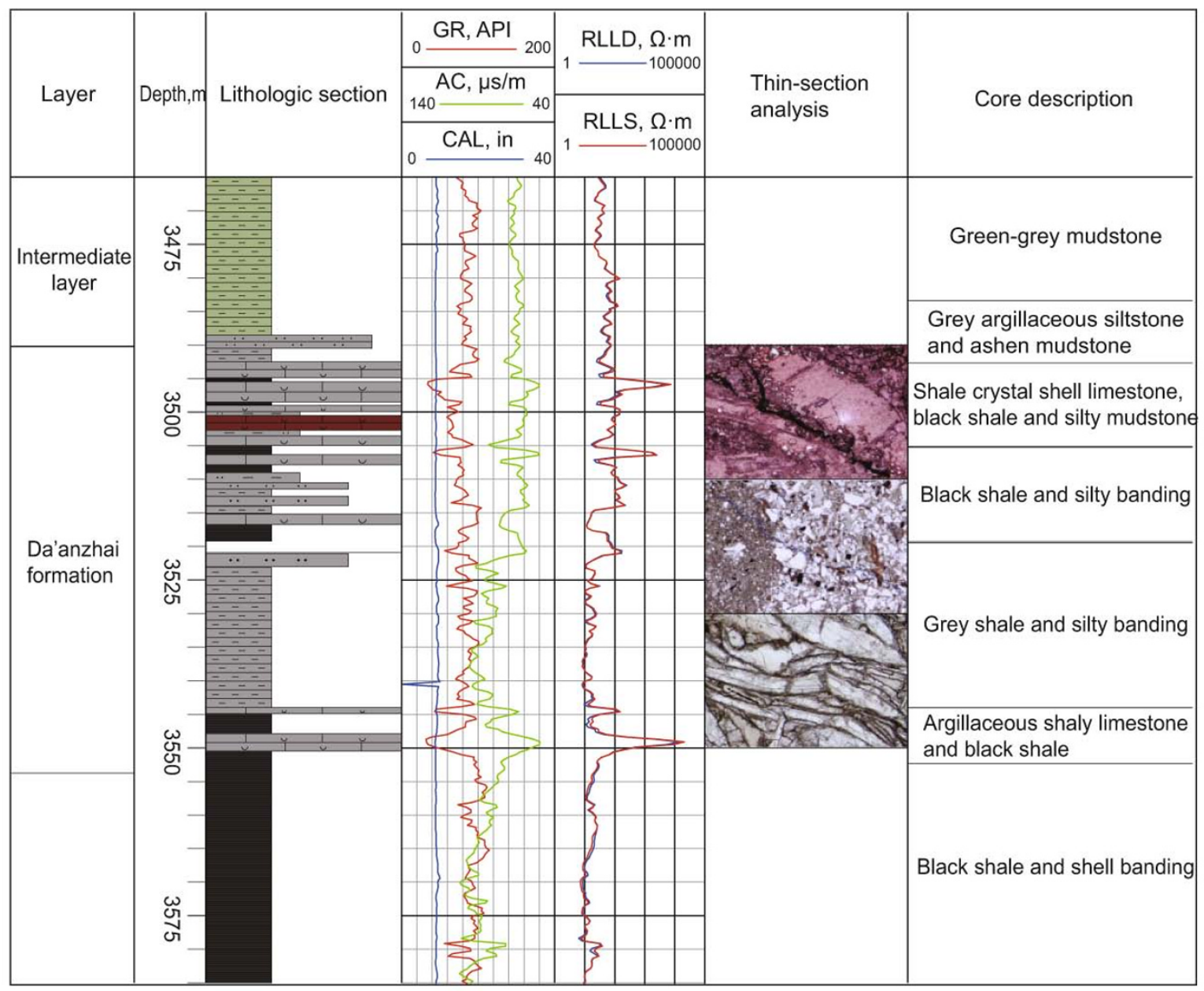

Fig. 1 Composite core column section of Longqian X well 
shale content than micrite shelly limestone. Petrological examination shows that the three cores are respectively micrite shelly limestone, silt to fine-grained quartz sandstone and shaly shelly limestone. There developed dissolved pores and a number of unfilled reticular micro-fractures in the shells in the micritic shelly limestone. The silty to fine-grained quartz sandstone has unfilled tortuous micro-fractures. The storage space in the muddy shelly limestone is mainly horizontal structural fractures, and the lamellar-shaped shells are mostly in a matrix of shale. The matrix porosity of the shelly limestone reservoir is generally less than $3 \%$, mainly between $0.5 \%$ and $2 \%$, and its permeability is largely between 0.001 and $1 \mathrm{mD}$, representing ultra-low porosity and ultralow permeability. In addition, the reservoir logging responses show low natural gamma ray values, low neutron porosity and acoustic slowness, relatively high lithology density and resistivity values. In the fractured interval, dual laterolog data shows medium-high resistivity values with a high resistivity background.

The Da'anzhai reservoir, a "self-source" and "selfreservoir" type with fairly good source-reservoir-cap assemblage, is the main oil and gas producer in the Longgang region. The matrix porosity of the productive layers ranges between $1.3 \%$ and $3.0 \%$ in general. Core slice analysis shows that the reservoir rock developed many structural fractures and structural micro-fractures, which serve both as important storage space and seepage channels. Therefore, research on the properties of matrix pores and fractures and their combination relationship is the key to evaluating reservoir effectiveness.

\section{Experimental analysis of reservoir matrix pore structure characteristics}

\subsection{Experimental methods and conditions}

\section{1) Nuclear magnetic resonance (NMR) experiment}

20 core samples with porosity between $0.45 \%$ and $1.46 \%$, and permeability between 0.0004 and $1.7 \mathrm{mD}$ were chosen for the NMR experiments. The NMR measurements were carried out with the $2 \mathrm{MHz}$ NMR core analyzer from Niumai Electronic Technology Company. The test temperature was
$25{ }^{\circ} \mathrm{C}$, the waiting time and echo spacing were respectively $6 \mathrm{~s}$ and $0.3 \mathrm{~ms}$, and the echo number was 4,096. All the samples were measured under both the fully water saturated and bound water conditions. The bound water condition was obtained with the centrifugal method. The maximum centrifugal force was $5 \mathrm{MPa}$. The samples were centrifuged over two hours under constant low temperature until the core weight remained the same. Spectrum decomposition of the NMR transverse relaxation signal was implemented to analyze the morphology of the NMR $T_{2}$ spectrum and the corresponding characteristic parameters under both water saturated and bound water conditions.

\section{2) Mercury injection porosimetry}

The measurements were carried out with an AutoPore IV 9505 automatic high pressure mercury porosimeter from Micromeritics Instrument Corporation. The porosimeter settings were selected according to the porosity of the samples. First the low pressure mercury injection experiment was carried out (0.019 MPa-0.19 MPa). To avoid the effect of the contact oxidation of the mercury and samples on the experimental results, the high pressure mercury injection experiment ( $0.19 \mathrm{MPa}-198 \mathrm{MPa}$ ) was carried out immediately after the low pressure analysis. Mercury injection data at various pressures were recorded to determine the median pressure $P_{\mathrm{c}}$, displacement pressure $P_{\mathrm{d}}$, average pore throat radius, median, and sorting coefficient.

\subsection{Matrix pore structure analysis}

Matrix pore structure refers to the geometry, size, spatial distribution of pores and throats and their connection relationship in the matrix. It is an important indicator to show if matrix pores form effective storage space. Capillary pressure curves characterize the rock pore throat size and distribution characteristics, reflecting the fluid seepage capacity of the medium. The $T_{2}$ spectrum of NMR mainly reflects pore distribution and the movable fluid in pores. Based on the NMR and capillary pressure experiments, the rock matrix pore structure is divided into three types according to the analysis of curve shape and experimental data. The basic characteristics of the three types are shown in Table 1.

Table 1 Characteristic parameters of three types of pore structure

\begin{tabular}{cccccc}
\hline & $\begin{array}{c}\text { Displacement pressure, } \\
\text { MPa }\end{array}$ & $\begin{array}{c}\text { Median pressure, } \\
\text { MPa }\end{array}$ & $\begin{array}{c}\text { Maximum mercury } \\
\text { saturation, } \%\end{array}$ & $\begin{array}{c}\text { Pore throat radius, } \\
\mu \mathrm{m}\end{array}$ & $T_{2}$ NMR spectrum \\
\hline Type I & $2.25-4.08$ & $18.2-38.4$ & $74.7-84.4$ & $0.063-0.16$ & Double peaks and obvious signal of big pores \\
Type II & $4.41-7.12$ & $29.8-63.4$ & $58.6-80.8$ & $0.01-0.063$ & Double peaks and signals of small pores predominate \\
Type III & $13.02-20.37$ & $130.6-171.4$ & $51.2-56.4$ & $0.004-0.01$ & Single peak and mainly small pores \\
\hline
\end{tabular}

Type I matrix pore structure: The central platform of the mercury injection curve is long and flat, as shown in Fig. 2(a). With an average displacement pressure of $3.15 \mathrm{MPa}$, an average median pressure of $25.7 \mathrm{MPa}$, and relatively high maximum mercury saturation of $80 \%$, this type of matrix pore has fairly good seepage capacity. The matrix pore throat is uniformly distributed and well sorted. The pore throat radii are between $0.063-0.16 \mu \mathrm{m}$, the mean radius is 0.054 $\mu \mathrm{m}$, and the median radius is $0.034 \mu \mathrm{m}$ which is relatively large, indicating that the distribution of matrix pore throats is ideal and the pore structure is good. Fig. 2(b) shows that the NMR $T_{2}$ spectrums of the saturated and centrifuged rock 
show double peaks. After centrifuging, both the movable peak and the immovable peak drop significantly in amplitude, and the signal amplitude shows that small pores predominate, while the signal of big pores is also strong. In terms of the relaxation time, small pores fall between 0.1-40 ms, while big pores fall between 40-500 ms. With a clear signal of big pores, and concentrated pore radius, this type of reservoir has good seepage behavior.

Type II matrix pore structure: The intermediate platform section of the mercury injection curve becomes short and high, as shown in Fig. 3(a). With an average displacement pressure of $5.65 \mathrm{MPa}$, an average median pressure of 48.1 $\mathrm{MPa}$, and the maximum mercury saturation of about $74.3 \%$, this type of reservoir has average seepage capacity. The pore throat radii are between 0.01 and $0.063 \mu \mathrm{m}$, the average mean radius of the pore throats is $0.0416 \mu \mathrm{m}$, and the average median radius of the pore throats is about $0.0304 \mu \mathrm{m}$, indicating that the pore throats are small and the quality of the pore structure is average. Most of the $T_{2}$ NMR spectrum has a double-peak shape, as shown in Fig. 3(b), and the signal is significantly lower than that of type I reservoirs. In terms of the relaxation time, small pores fall between 0.1 and $70 \mathrm{~ms}$, while big pores fall in 70-3,000 ms, relatively long relaxation time suggesting that the pore radius spans a wide range, and this type of reservoir is poorer in seepage performance than type I reservoirs.

Type III matrix pore structure: The intermediate platform section of the mercury injection curve is hardly observed as shown in Fig. 4(a). The average displacement pressure is 16.4 $\mathrm{MPa}$, the average median pressure is $153 \mathrm{MPa}$, and the maximum mercury saturation is only about $56 \%$, indicating poor seepage capacity. With the pore throat radii between 0.004 and $0.01 \mu \mathrm{m}$, the mean radius drops down to 0.0103 $\mu \mathrm{m}$, and the median radius decreases to $0.0047 \mu \mathrm{m}$, showing very fine pore throats and poor pore structure. The NMR $T_{2}$ spectrum presents a single peak shape, which indicates that the matrix pores are small and their size range is narrow. In addition, the movable fluid signal disappears completely, as shown in Fig. 4(b), indicating that this type of reservoir has very poor matrix pore seepage capacity.

Low porosity low permeability carbonate reservoirs have complex pore structure, fine pore throat, and micro fractures develop, making the occurrence of fluid in the pores very complex. For type I pore structure, the maximum mercury saturation of the samples is between $75 \%$ and $84 \%$ and the fluid signals of centrifuged samples decrease significantly. For type II pore structure, the maximum mercury saturation of the samples ranges between $59 \%$ and $81 \%$ and the seepage capacity is poor. For type III pore structure, the maximum mercury saturation of samples falls between $51 \%$ and $56 \%$, the mercury ejection efficiency is commonly between $20 \%$ and $30 \%$, and the fluid signal of centrifuged samples is little different from the saturated ones.

The mercury injection experiment and the NMR experiment show that the characteristics of pore occurrence determine the seepage capacity of matrix pores. From type III to I, the storage capacity of matrix pores increases. Although under normal conditions the measured matrix porosity and permeability are very low, (even for the type I matrix pore structure, the porosity is below $1.46 \%$, and the permeability is below $1.7 \mathrm{mD}$, which cannot exactly display the actual seepage capacity of the matrix pores.) under reservoir conditions the matrix pores can still be effective fluid storage space with certain seepage capacity.

\section{Effectiveness analysis of matrix pores}

The percentage of mobile fluid $S_{\mathrm{m}}$ is defined as the ratio of the accumulated amplitude of $T_{2}$ which is greater than $T_{2}$ cutoff, to the total accumulated amplitude. The movable fluid porosity $\phi_{\mathrm{m}}$ is the product of the percentage of mobile fluid and the rock porosity. The percentage of mobile fluid of the 20 selected cores is between $25 \%$ and $51 \%$, with an average of $39 \%$, and the movable fluid porosity is between $0.18 \%$ and $0.54 \%$, with an average of $0.31 \%$, indicating that the movable fluid content of tight carbonate reservoirs in this area is overall low. Moreover, the movable fluid porosity and porosity show a good positive correlation as shown in Fig. 5.

The capillary pressure experiment shows that the pore throat radii of this reservoir are small overall but the pore throat radii of type I matrix pore structure range mainly above $0.063 \mu \mathrm{m}$. Taking $0.063 \mu \mathrm{m}$ as the critical value that fluid

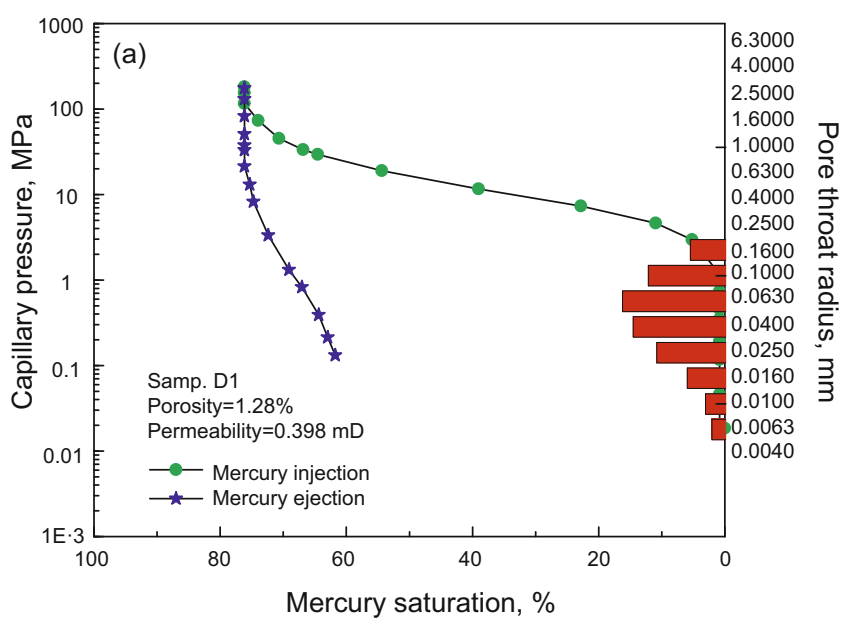

(a) Mercury injection capillary pressure curve

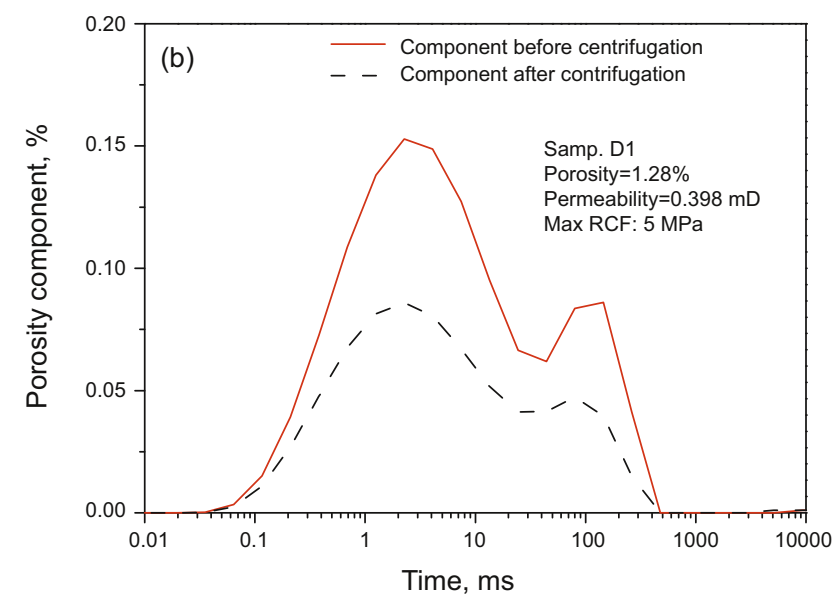

(b) Nuclear magnetic resonance curve

Fig. 2 Matrix pore structure of type I 


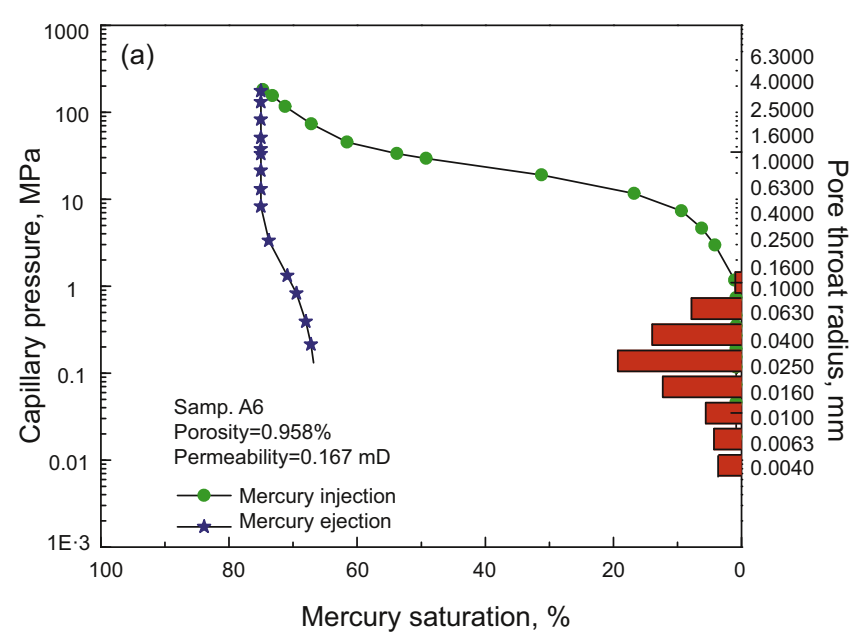

(a) Mercury injection capillary pressure curve

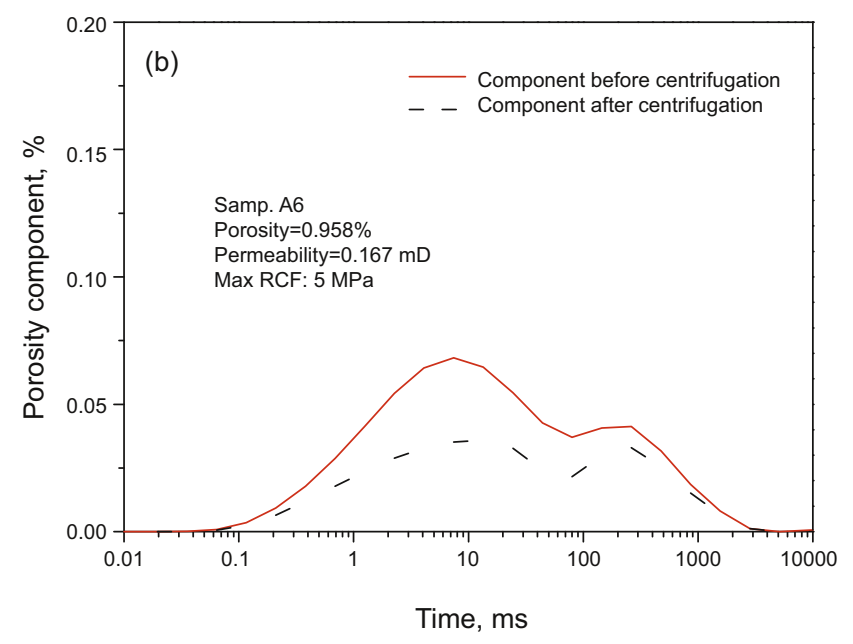

(b) Nuclear magnetic resonance curve

Fig. 3 Matrix pore structure of type II

can flow through, the seepage space percentage is defined as the percentage of pore throat volume with pore throat radius greater than or equal to $0.063 \mu \mathrm{m}$, to the total pore throat volume. Fig. 6 shows that the pore throat volume of seepage flow and porosity show a good positive correlation. The NMR $T_{2}$ spectrum and the capillary pressure experiment show that, in ultra-low porosity and ultra-low permeability reservoirs, if the matrix pores are relatively well developed and the pore structure is good (such as type I), the matrix pores can become effective storage space for oil and gas, and play an important role in the low and stable oil and gas production.

\section{Evaluation of fracture effectiveness}

\subsection{Calculation of fracture parameters}

\section{1) Identification of fracture occurrence}

It is effective to identify fractures with dual laterolog data (Liu et al, 2009; Deng et al, 2006). In view of different fracture occurrences, the fractures are divided into three states, namely low angle fractures $\left(0-50^{\circ}\right)$, intermediate angle fractures $\left(50^{\circ}-75^{\circ}\right)$ and high angle fractures $\left(75^{\circ}-90^{\circ}\right)$. According to the resistivity of the deep and shallow laterolog, fracture occurrence can be identified by the following

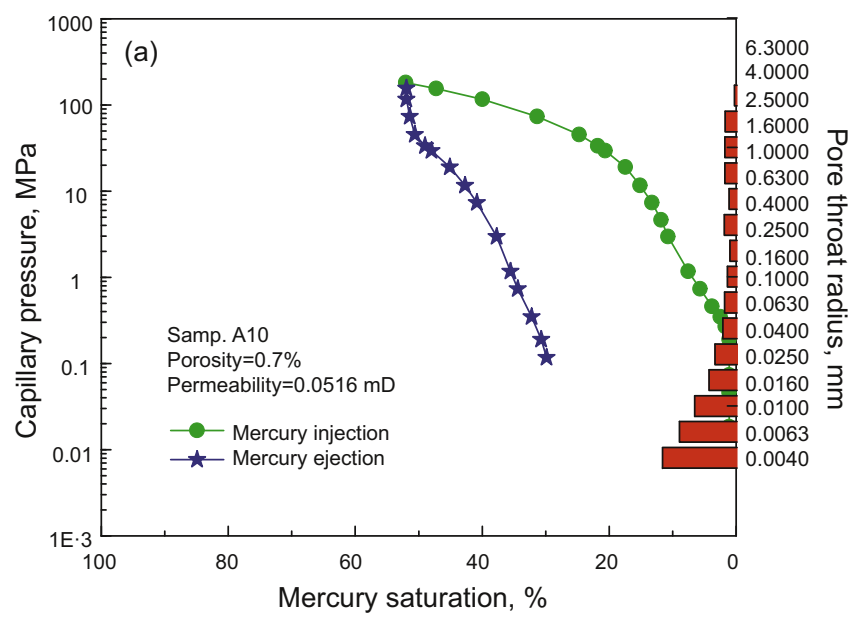

(a) Mercury injection capillary pressure curve

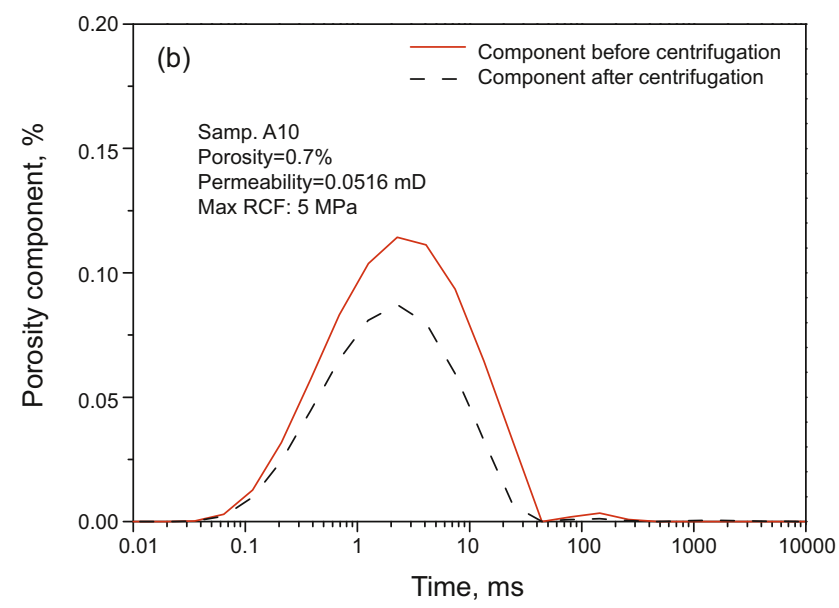

(b) Nuclear magnetic resonance curve

Fig. 4 Matrix pore structure of type III

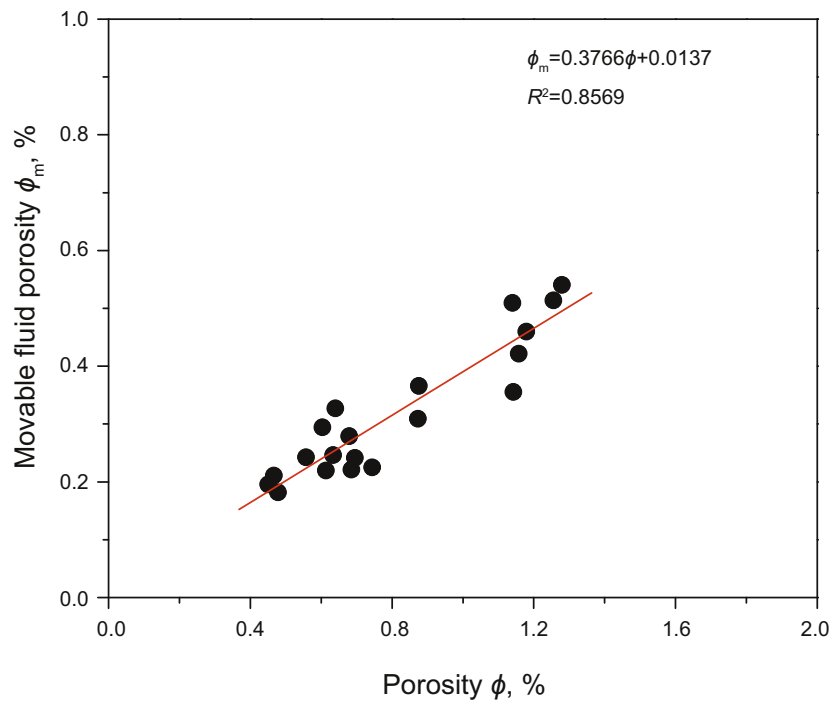

Fig. 5 Movable fluid \& porosity relationship

equation (Li et al, 1996)

$$
Y=\left(R_{\mathrm{LLD}}-R_{\mathrm{LLS}}\right) / \sqrt{R_{\mathrm{LLD}} \cdot R_{\mathrm{LLS}}}
$$




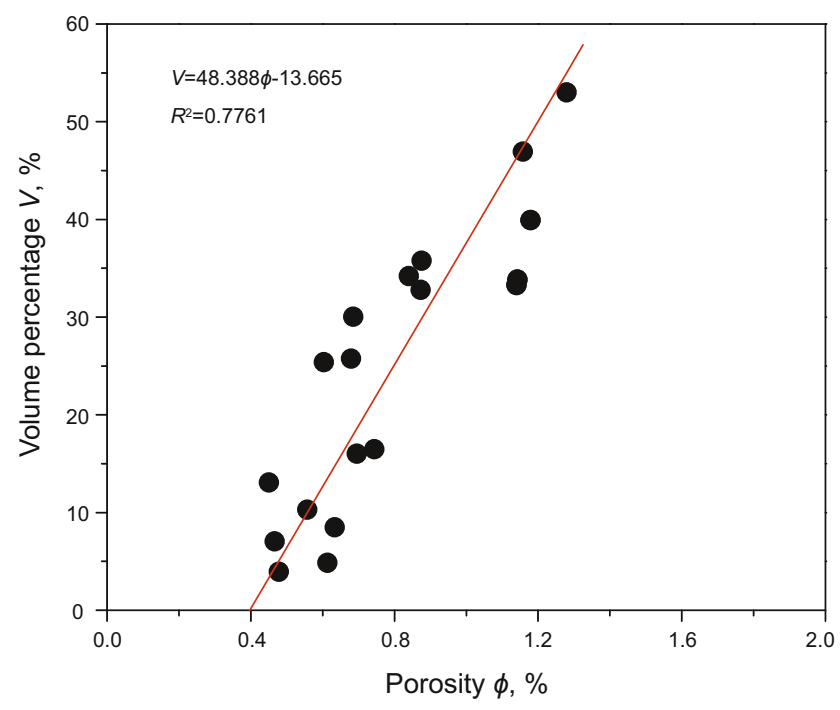

Fig. 6 Pore volume $(\geq 0.063 \mu \mathrm{m}) \&$ porosity relationship

where, $R_{\mathrm{LLD}}$ and $R_{\mathrm{LLS}}$ are respectively the deep and shallow laterolog resistivity, $Y$ is the fracture state parameter. If the value of $Y$ is lower than 0 , the fracture has a low angle. If the value of $Y$ is between 0 and 0.1 , the fracture has an intermediate angle. The value of $Y$ greater than 0.1 indicates a high angle.

\section{2) Calculation model of fracture porosity}

Using the flat panel fracture model (Deng et al, 2006), under particular borehole conditions, according to the matrix rock resistivity distribution, a fast calculation model for the dual laterolog responses to fractures was established, and fast inversion of the fracture porosity was performed with the least square principle.

a) The matrix rock resistivity $\left(R_{\mathrm{b}}\right)$ is greater than $1,000 \Omega \cdot \mathrm{m}$
Dual laterolog apparent resistivity can be approximately expressed as

$$
\begin{aligned}
& 1 / R_{\mathrm{LLD}}=D_{i, 0} \cdot\left(\sigma_{\mathrm{f}} \cdot \phi_{\mathrm{f}}\right)^{D_{i, 1}} \cdot R_{\mathrm{b}}+D_{i, 2} \cdot\left(\sigma_{\mathrm{f}} \cdot \phi_{\mathrm{f}}\right)^{D_{i, 3}} \\
& 1 / R_{\mathrm{LLS}}=S_{i, 0} \cdot\left(\sigma_{\mathrm{f}} \cdot \phi_{\mathrm{f}}\right)^{S_{i, 1}} \cdot R_{\mathrm{b}}+S_{i, 2} \cdot\left(\sigma_{\mathrm{f}} \cdot \phi_{\mathrm{f}}\right)^{S_{i, 3}}
\end{aligned}
$$

where $D_{i, j}, S_{i, j}(i=1,2,3)$ are three different values according to the three states of fractures including low angle, intermediate angle and high angle.

b) The matrix rock resistivity $\left(R_{\mathrm{b}}\right)$ is between $50 \Omega \cdot \mathrm{m}$ and $1,000 \Omega \cdot \mathrm{m}$

$$
\begin{aligned}
& \log R_{\mathrm{LLD}}=D_{2} \cdot \log ^{2} R_{\mathrm{b}}+D_{1} \cdot \log R_{\mathrm{b}}+D_{0} \\
& \log R_{\mathrm{LLS}}=S_{2} \cdot \log ^{2} R_{\mathrm{b}}+S_{1} \cdot \log R_{\mathrm{b}}+S_{0}
\end{aligned}
$$

where $D_{i}, S_{i}(i=0,1,2)$ are coefficients, the values are respectively

$$
\begin{aligned}
& D_{i}=A_{i, 3} \log ^{3}\left(\phi_{\mathrm{f}} \sigma_{\mathrm{f}}\right)+A_{i, 2} \log ^{2}\left(\phi_{\mathrm{f}} \sigma_{\mathrm{f}}\right)+A_{i, 1} \log \left(\phi_{\mathrm{f}} \sigma_{\mathrm{f}}\right)+A_{i, 0} \\
& S_{i}=B_{i, 3} \log ^{3}\left(\phi_{\mathrm{f}} \sigma_{\mathrm{f}}\right)+B_{i, 2} \log ^{2}\left(\phi_{\mathrm{f}} \sigma_{\mathrm{f}}\right)+B_{i, 1} \log \left(\phi_{\mathrm{f}} \sigma_{\mathrm{f}}\right)+B_{i, 0}
\end{aligned}
$$

where $A_{i, j}, B_{i, j}$ are three different values according to the three occurrence states of fractures.

c) Tests on the effectiveness of the calculated fracture porosity

The effectiveness of the method was examined by comparing the fracture porosity (POR2-DLL), calculated from dual laterolog data and formation microimaging (FMI) logging fracture porosity (POR2-FMI) as shown in Fig. 7. PORT is the total porosity calculated from FMI. RLLD is the deep laterolog resistivity. RLLS is the shallow laterolog

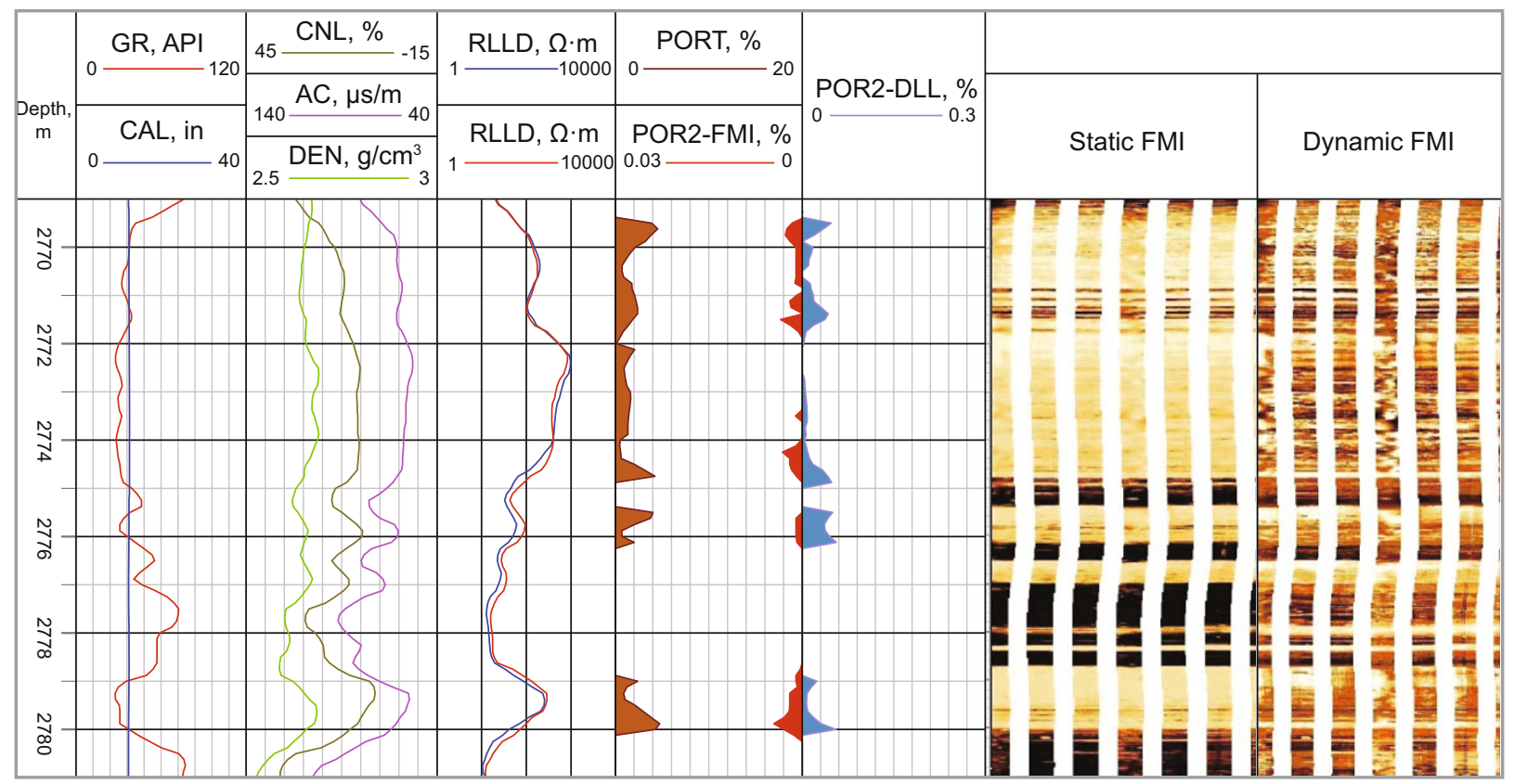

Fig. 7 Comparison between dual laterolog fracture porosity and FMI fracture porosity 
resistivity. CNL is the compensated neutron $\log$. AC is the acoustic slowness log. DEN is the compensated density log. CAL is the diameter of borehole and GR is natural gamma ray $\log$. It shows that the variations of the calculated fracture porosity from dual laterolog data are in good agreement with the trends of fracture porosity from FMI, but the actual values are 10 times apart, caused by different logging mechanisms. FMI logging provides the information of fracture porosity of the borehole wall, while the dual laterolog method reflects the macroscopic fracture porosity within the deeper detection range (Deng et al, 2006).

\subsection{Division of fracture pore development level}

Because of tectonic stress, fractures usually develop in the form of fracture belts with complex heterogeneity. Under similar geological background conditions, fractures have similar type or occurrence characteristics, and fracture porosity basically presents a normal distribution. Accordingly the cumulative trend of fracture porosity exhibits a linear relationship. The heterogeneity of fractures can be shown by the multiple normal distributions and peak combinations in fracture porosity histograms. However, in different formation conditions, the development of fracture porosity may have similar characteristics, together with the processing errors of the actual logs, the normal distributions could become nonstandard and the peaks are not obvious.

Fig. 8 presents the frequency statistics of fracture porosity of 15 wells in the Da'anzhai tight carbonate reservoirs (60 $\mathrm{m}$ in average thickness). The fracture porosity is basically lower than $0.15 \%$ and results from a combination of four evident nonstandard normal distributions, suggesting that the reservoirs have strong heterogeneity, with a variety of states or types of fractures in this target area. Fig. 9 shows the fracture porosity accumulation curve which can be approximately considered as a combination of multiple linear distributions. It indicates the obvious differences of fracture development under different conditions. According to the fracture porosity accumulation shown in Fig. 9, on the basis of the theory that the same fracture development level has the same trend

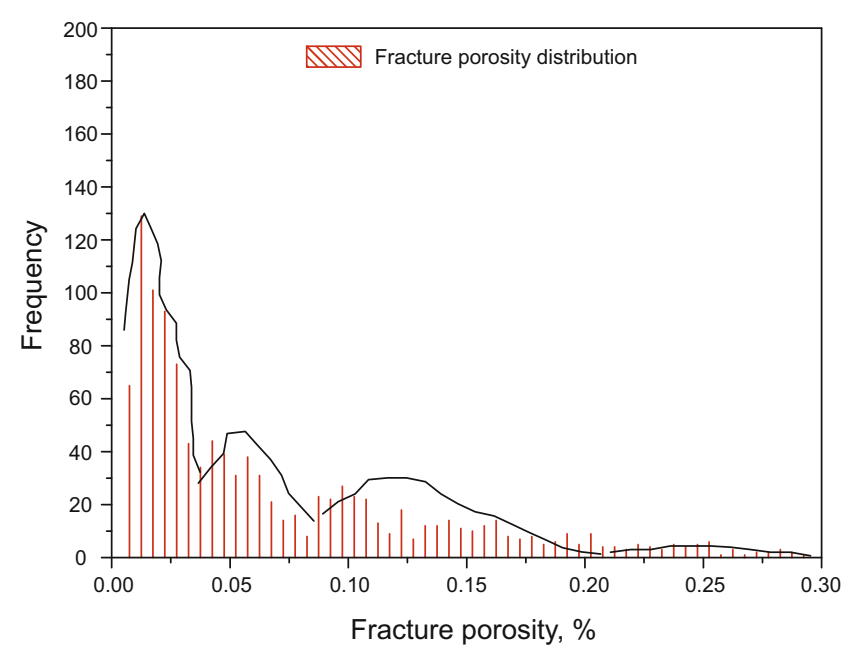

Fig. 8 Fracture porosity distribution histogram envelope features, the inflection point of the cumulative trend line is used to classify the development degree of fractures. The figure shows that the fracture development can be basically divided into four classes. However, considering the very small number of reservoirs with fracture porosity greater than $0.22 \%$, a three-class method is adopted as $\phi_{\mathrm{f}}>0.085 \%$, $0.035 \%<\phi_{\mathrm{f}}<0.085 \%$ and $\phi_{\mathrm{f}}<0.035 \%$.

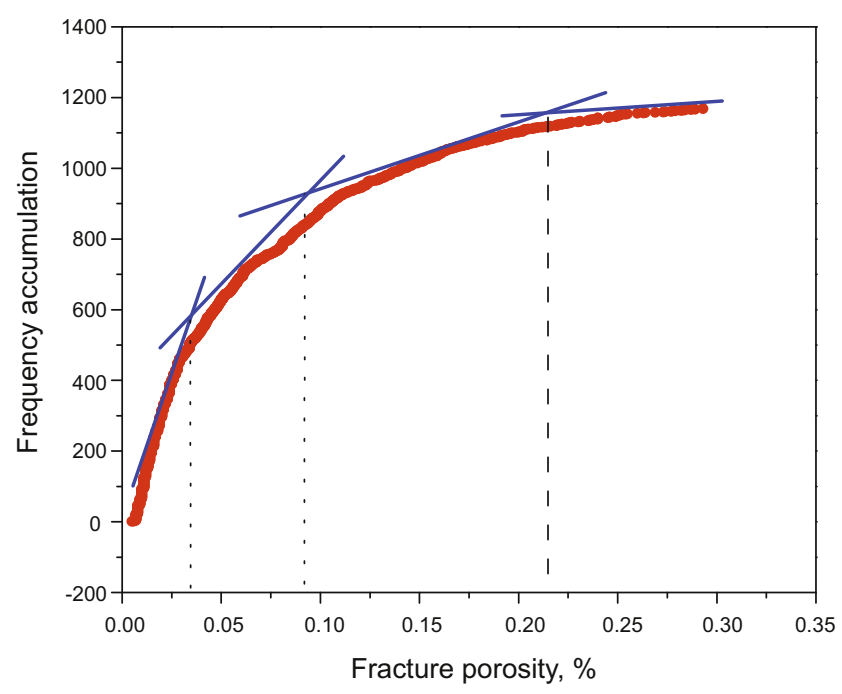

Fig. 9 Fracture porosity classification principle

\section{Comprehensive evaluation of reservoir effectiveness}

\subsection{Reservoir level classification basis}

The development level of matrix pores and fractures determine the tight reservoir storage and seepage capacity, so with good match of porosity and permeability and good oil source, tight reservoirs can be of commercial value (Li et al, 2011). According to the capacity classification method for fracture-cavity carbonate reservoirs (Yang et al, 2010) and the dynamic production data, the reservoirs are classified as follows:

Class-A: Intermediate-high production reservoirs-for a kilometer deep well, its stable daily oil production is greater than 5 tons or daily gas production is greater than 30,000 cubic meters.

Class-B: Low production reservoir-for a kilometer deep well, its stable daily oil production is between 1 ton and 5 tons or daily gas production is between 3,000 cubic meters and 30,000 cubic meters.

Class-C: Ultra-low production reservoir-for a kilometer deep well, its stable daily oil production is less than 1 ton or daily gas production is less than 3,000 cubic meters.

\subsection{Reservoir level classification method}

Based on the classification of matrix porosity and fracture porosity, and by utilizing logging responses, reservoir storage 
space type, the mercury injection experiment, the NMR experiment and the actual production test, the tight carbonate reservoirs in Da'anzhai are classified as follows.

First class reservoir (I): matrix pores and fractures are well developed, and the storage space is fractured-porosity type. This type of reservoir has high neutron porosity, high acoustic slowness and low density response. The dual laterolog data shows no difference or small positive difference, and the resistivity is low and only greater than the shale resistivity. The mercury injection curve shows low displacement pressure, low median pressure, small-to-medium but uniformly distributed pore throat radii, and good sorting, which indicates that the matrix pores have good connectivity. The NMR $T_{2}$ spectrum shows an obvious bimodal structure, and the signal of big pores is obvious, indicating that the matrix pores have good seepage capacity. This type of reservoir can be classified as class-A productivity.

Second class reservoirs (II): matrix pores and fractures are less developed than first class reservoirs and the storage space is usually poor fracture-pore or poor fracture type. The three porosity logs are similar in a shape with gentle change. The deep and shallow laterolog have obvious positive differences and the resistivities are medium-low against high resistivity background. The mercury injection curve shows mediumhigh displacement pressure and median pressure, medium pore throat radius, average sorting, suggesting that the matrix pore connectivity is poor. The NMR $T_{2}$ spectrum mostly shows a bimodal structure. The signal of small pores becomes predominant, indicating that the reservoir mainly contains small pores and can be classified as class-B productivity.

Third class reservoirs (III): with poorly developed matrix pores and fractures, this type of reservoir is hardly effective. The logging responses show low natural gamma, acoustic slowness and neutron porosity, high lithology density and high dual laterolog resistivity even with peak shape. The mercury injection curve show extremely high displacement pressure value and median pressure value and extremely fine pore throat radii, suggesting very poor connectivity of matrix pores. The NMR $T_{2}$ spectrum presents an obvious unimodal form. Almost mere signals of small pores occur. This type of reservoir, very poor in seepage ability, corresponds to the Class-C productivity.

\subsection{Reservoir level classification standard}

Based on the classification principle of productivity level of carbonate reservoirs, deep lateral resistivity $\left(R_{\mathrm{LLD}}\right)$, fracture porosity $\left(\phi_{\mathrm{f}}\right)$ and matrix porosity $\left(\phi_{\mathrm{b}}\right)$ are selected as the sensitive parameters to classify the reservoir level. The analysis of 59 layers from 10 test wells in this region shows a good match between the reservoir productivity level of A, B, $\mathrm{C}$ and the matrix porosity, the fracture porosity and the deep laterolog resistivity. The coincidence rate reaches as high as $90 \%$, as shown in Fig. 10 and Fig. 11. Thus, we determined the value ranges of matrix porosity, fracture porosity and deep lateral logging for the three-class productivity reservoirs, as shown in Table 2, in which the matrix porosity is obtained from neutron-density measurements.

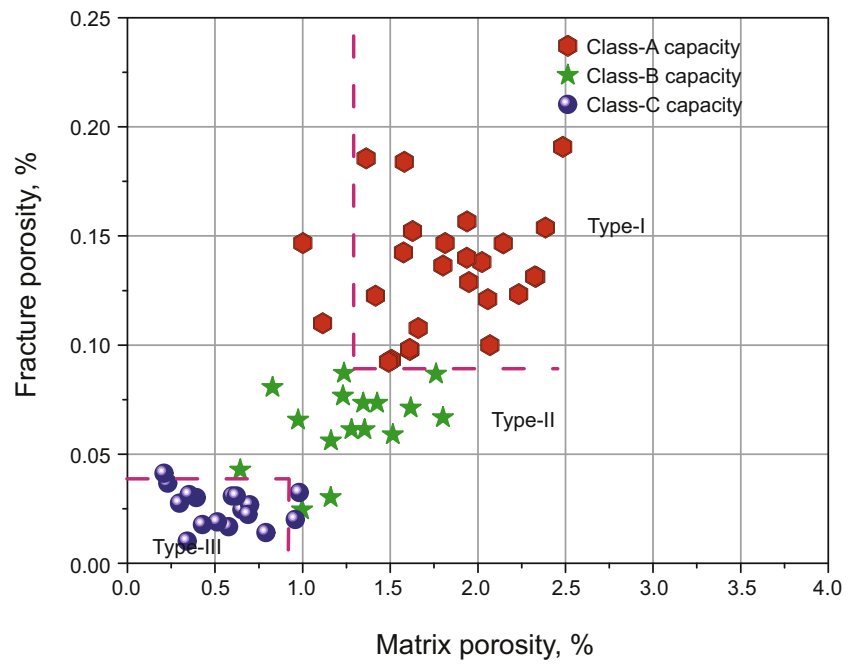

Fig. 10 Matrix porosity and fracture porosity cross-plot

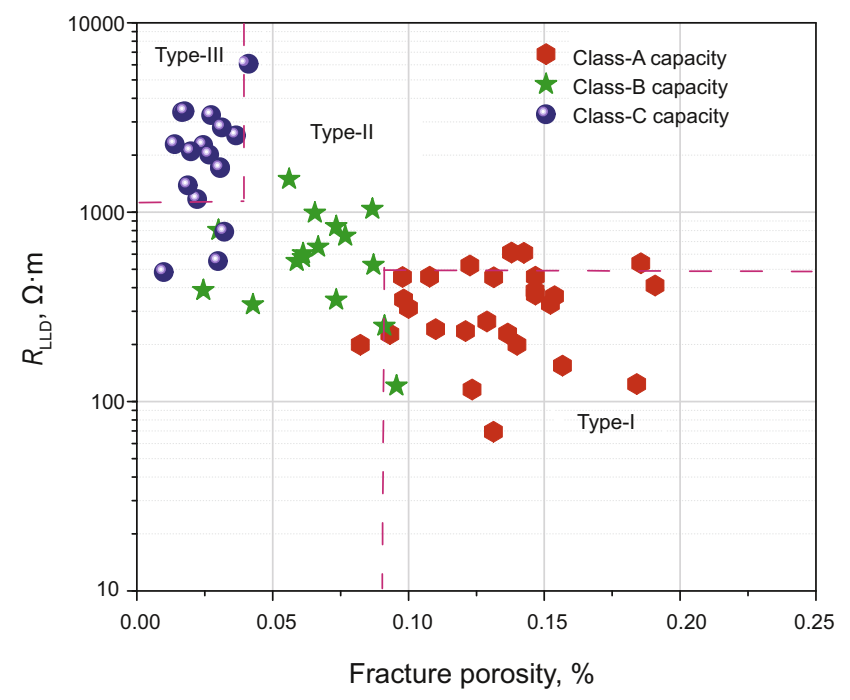

Fig. 11 Fracture porosity and deep resistivity cross-plot

Table 2 Reservoir level classification standard

\begin{tabular}{cccc}
\hline $\begin{array}{c}\text { Reservoir } \\
\text { level }\end{array}$ & $\begin{array}{c}\text { Matrix porosity, } \\
\phi_{\mathrm{b}}\end{array}$ & $\begin{array}{c}\text { Fracture porosity, } \\
\phi_{\mathrm{f}}\end{array}$ & $\begin{array}{c}\text { Deep lateral resistivity, } \\
R_{\mathrm{LLD}}\end{array}$ \\
\hline I class & $1.3 \%-$ & $0.085 \%-$ & $<500 \Omega \cdot \mathrm{m}$ \\
II class & $0.8 \%-1.7 \%$ & $>0.0035 \%$ & $300-1100 \Omega \cdot \mathrm{m}$ \\
III class & $<0.8 \%$ & $<0.035 \%$ & $>1100 \Omega \cdot \mathrm{m}$ \\
\hline
\end{tabular}

\section{Case study}

Fig. 12 is the comprehensive interpretation results of Longqian X well. Layer No.1 (3,174.6-3,175.7 m) has a fracture porosity of about $0.023 \%$, and an average matrix porosity of $0.38 \%$. Layer No.2 (3,178.6-3,190.1 m) has a fracture porosity of $0.011 \%$, with no fractures developed, and an average matrix porosity of $0.5 \%$, and an average deep lateral resistivity of over $3,000 \Omega \cdot \mathrm{m}$. Layer No.3 $(3,195.7$ $3,197.4 \mathrm{~m}$ ) has a fracture porosity of $0.021 \%$, and an average 


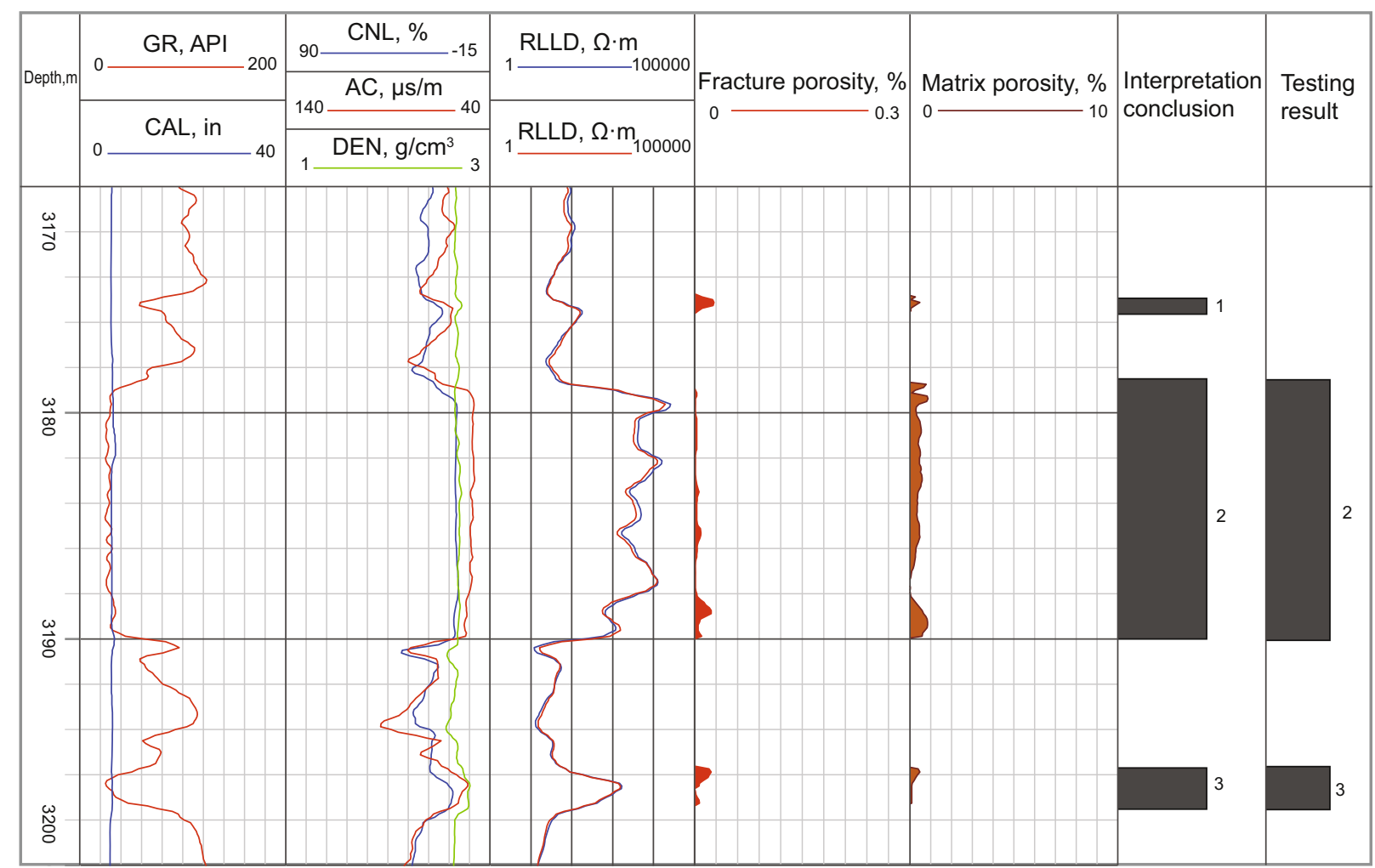

Fig. 12 Reservoir classification interpretation of Longqian $X$ well

matrix porosity of $0.3 \%$. All the three layers, with poorly developed fractures and matrix pores, are interpreted as Class III according to the reservoir comprehensive classification standard. According to the productivity level standard, they belong to Class $\mathrm{C}$ reservoirs of extremely low production. An oil production test yielded $0.1 \mathrm{t}$ oil a day, and a trace of gas.

The Longqian $\mathrm{H} 1$ well is the first horizontal oil well in the Longgang area, with its trajectory near the fault. Most sections of the well are in the bed of interest except for some sections in shale because of the influence of the fault. Fig. 13 shows the interpretation results of the Longqian H1 well. Matrix porosity $\phi_{\mathrm{b}}$ is relatively high, between $1.3 \%$ and $3.1 \%$. So it is hard to classify the reservoir according to matrix porosity alone, $R_{\mathrm{LLD}}$ and fracture porosity should be considered to make a comprehensive evaluation.

The criteria mentioned above are used to evaluate Layer No.1, Layer No.1-2 with $R_{\text {LLD }}$ of $130-960 \Omega \cdot \mathrm{m}$, and $\phi_{\mathrm{f}}$, of $0.06 \%-0.14 \%$, is identified as class I reservoirs; while Layer No.1-1 and 1-2 with high $\phi_{\mathrm{b}}$, low $\phi_{\mathrm{f}}$ and high $R_{\mathrm{LLD}}$, are determined as class III reservoirs. Formation testing of Layer No.1 shows that it is an oil layer. Layer No.2-2 and 2-4 with $R_{\text {LLD }}$ of $700-1,100 \Omega \cdot \mathrm{m}$, and relatively high $\phi_{\mathrm{f}}$ of $0.07 \%$ are identified as class II reservoirs; while Layer No.2-1 and 2-3 fit the features of class III reservoirs in terms of $R_{\mathrm{LLD}}, \phi_{\mathrm{f}}$ and $\phi_{\mathrm{b}}$. Formation testing shows that Layer No.2 is dry. In the interpretation of Layer No.3 and No.5, after deducting some high resistivity interbeds, the rest Layers No.3-1, 3-2, 5-1 to 5-5 all show the characteristics of class I reservoirs, and formation testing confirms they are all oil layers. Layer No.4 with very high $R_{\text {LLD }}$ of over $1,000 \Omega \cdot \mathrm{m}$ on average, and very low fracture porosity, is determined as class III, while formation testing confirms it is a dry layer.

\section{Conclusions}

1) Though Da'anzhai carbonate reservoirs are low in matrix porosity and matrix permeability, the matrix pores can be major storage space when the matrix porosity and the pore-throat parameters reach a certain range. The movable fluid in the matrix pores makes an important contribution to the reserves and long-term low and stable production of effective reservoirs.

2) Fractures, serving as both essential storage space and seepage channels for fluid, are an important index of the reservoir productivity. It is effective to calculate fracture parameters of tight reservoirs by fast inversion of dual laterolog data. Based on the distribution of regional fracture porosity, the cumulative curve and the heterogeneity of reservoirs, a classification standard of three classes of fracture development was set up.

3) The dual storage spaces of matrix-pores and fractures are important factors affecting the reservoir effectiveness and productivity level. On the basis of the well and production tests, the classification criteria of the Da'anzhai tight carbonate reservoirs were determined through multiple intersection analysis of the matrix porosity, fracture porosity and deep laterolog resistivity. The classification results are in good agreement with the production results.

4) In the evaluation of the effectiveness of tight carbonate reservoirs of extremely-low porosity and permeability, the integrated evaluation of matrix pore effectiveness and fracture pore effectiveness can improve prediction accuracy. 


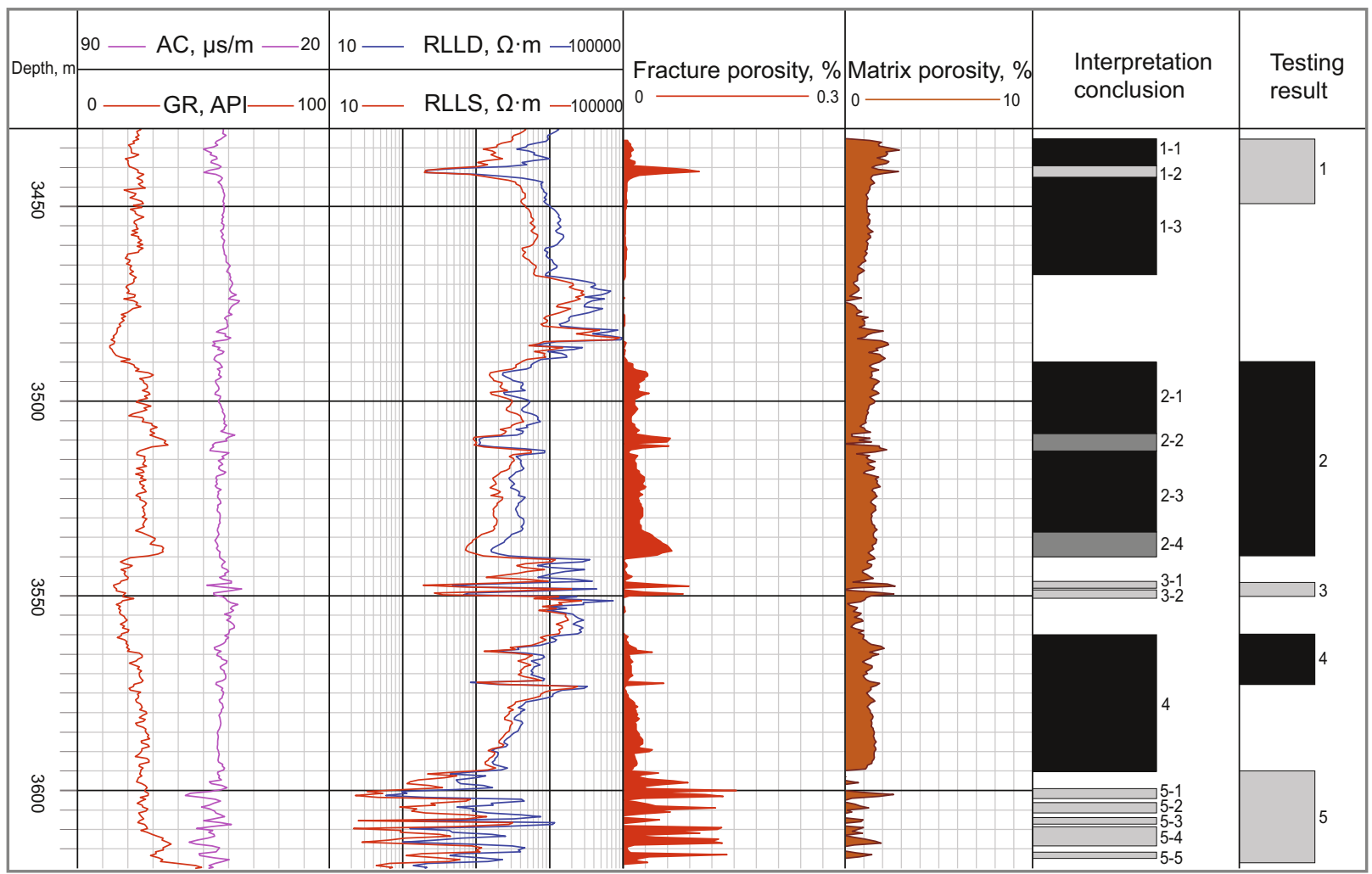

Fig. 13 Reservoir classification interpretation of Longqian H1 well

\section{Acknowledgements}

This study is co-funded by the National Natural Science Foundation of China (No.41174009) and National Major Science \& Technology Projects of China (Nos.2011ZX05020, 2011ZX05035, 2011ZX05009, 2011ZX05007). We would like to express our sincere appreciation to our respected Professor Mo Xuanxue, a geologist and member of the Chinese Academy of Sciences, for his instructive guidance.

\section{References}

Asgari A A and Sobhi G A. A fully integrated approach for the development of rock type characterization, in a Middle East giant carbonate reservoir. Journal of Geophysics and Engineering. 2006. 3(3): $260-270$

Bust V K, Oletu J U and Worthington P F. The challenges for carbonate petrophysics in petroleum resource estimation. SPE Reservoir Evaluation \& Engineering. 2011. 14(1): 25-34 (SPE 142819)

Chekani $\mathrm{M}$ and Kharrat R. An integrated reservoir characterization analysis in a carbonate reservoir: A case study. Petroleum Science and Technology. 2012. 30(14): 1468-1485

Deng S G, Wang X C, Zou D J, et al. Interpreting dual laterolog fracture data in fractured carbonate formation. Journal of China University of Geosciences. 2006. 17(2): 168-172

Guerriero V, Mazzoli S, Iannace A, et al. A permeability model for naturally fractured carbonate reservoirs. Marine and Petroleum Geology. 2012. 40(5): 115-134

Guo J C, Nie R S and Jia Y L. Dual permeability flow behavior for modeling horizontal well production in fractured-vuggy carbonate reservoirs. Journal of Hydrology. 2012. 464-465: 281-293

Kim K, Lee Y, Hwang S, et al. Improved capillary pressure model considering dual-pore geometry system in carbonate reservoirs. Journal of Petroleum Science and Engineering. 2011. 78(3-4): 601608

Kuz'min V A and Skibitskaya N A. Studies of the anisotropy of the microstructure of oil and gas reservoir rocks by electron microscopy methods. Journal of Surface Investigation: X-Ray Synchrotron and Neutron Techniques. 2012. 6(1): 187-193

Lamarche J, Lavenu A P C, Gauthier B D M, et al. Relationships between fracture patterns, geodynamics and mechanical stratigraphy in carbonates (South-East Basin, France). Tectonophysics. 2012. 581(SI): 231-245

Li F Y, Di B R, Wei J X, et al. A method for estimating the width of carbonate fracture-cavern bodies. Chinese Journal of Geophysics (Chinese Edition). 2012. 55(2): 631-636 (in Chinese)

Li S J, Xiao Y W, Wang H M, et al. Mathematical model of dual laterolog response to fracture and quantitative interpretation of fracture porosity. Chinese Journal of Geophysics. 1996. 39(6): 845-852 (in Chinese)

Li Y, Li B Z, Hu Y L, et al. Classification of carbonate gas condensate reservoirs using well test and production data analyses. Petroleum Science. 2011. 8(1): 70-78

Liang D G, Ran L H, Dai D S, et al. A re-recognition of the prospecting potential of Jurassic large-area and non-conventional oils in the central-northern Sichuan Basin. Acta Petrolei Sinica. 2011. 32(1): 8-17 (in Chinese)

Liu R L, Li N, Feng Q F, et al. Application of the triple porosity model in well-log effectiveness estimation of the carbonate reservoir in Tarim Oilfield. Journal of Petroleum Science and Engineering. 2009. 68(2): 40-46

Mai A and Kantzas A. Porosity distributions in carbonate reservoirs using low-field NMR. Journal of Canadian Petroleum Technology. 2007. 46(7): 30-36

Marathe R, Turner M L and Fogden A. Pore-scale distribution of crude 
oil wettability in carbonate rocks. Energy \& Fuels. 2012. 26(10): 6268-6281

Mohammadlou M H, Mørk M B and Langeland H. Integrated permeability analysis in tight and brecciated carbonate reservoir. SPE Reservoir Evaluation \& Engineering. 2012. 15(6): 624-635 (SPE 131593)

Pezard P A and Anderson R N. In situ measurements of electrical resistivity, formation anisotropy, and tectonic context. SPWLA 31st Annual Logging Symposium, 24-27 June, 1990, Lafayette Louisiana (SPWLA M)

Schoenfelder W, Gläser H R, Mitreiter I, et al. Two-dimensional NMR relaxometry study of pore space characteristics of carbonate rocks from a Permian aquifer. Journal of Applied Geophysics. 2008. 65(1): 21-29

Sibbit A M and Faivre O. The dual laterolog response in fractured rocks. SPWLA 26th Annual Logging Symposium, 17-20 June 1985, Dallas Texas (SPWLA T)

Tsakiroglou C D, Ioannidis M A, Amirtharaj E, et al. A new approach for the characterization of the pore structure of dual porosity rocks.
Chemical Engineering Science. 2009. 64(5): 847-859

Wang H Y, Sun S Z, Yang H J, et al. The influence of pore structure on P-\& S-wave velocities in complex carbonate reservoirs with secondary storage space. Petroleum Science. 2011. 8(4): 394-405

Westphal H, Surholt I, Kiesl C, et al. NMR measurements in carbonate rocks: Problems and an approach to a solution. Pure and Applied Geophysics. 2005. 162(3): 549-570

$\mathrm{Xu} \mathrm{X}$, Wei G Q and Yang Z M. The productivity calculation method of a carbonate reservoir. Petroleum Science and Technology. 2013. 31(14): 301-309

Yang H J, Sun S Z, Cai L L, et al. A new method of formation evaluation for fractured and caved carbonate reservoirs: A case study from the Lundong area, Tarim Basin, China. Petroleum Science. 2011. 8(4): 446-454

Yang W J, Xiao C W, Liu L F, et al. Logging evaluation of the Ordovician carbonate reservoir beds in the Lungudong region, Tarim Basin. Acta Geologica Sinica (English Edition). 2010. 84(5): 11411156

(Edited by Hao Jie) 